\title{
Designing Plasmonic Gratings with Transformation Optics
}

\author{
Matthias Kraft, ${ }^{1, *}$ Yu Luo, ${ }^{2, \dagger}$ S. A. Maier, ${ }^{1}$ and J. B. Pendry ${ }^{1}$ \\ ${ }^{1}$ Department of Physics, The Blackett Laboratory, Imperial College London, \\ London SW7 2AZ, United Kingdom \\ ${ }^{2}$ Centre for OptoElectronics and Biophotonics (OPTIMUS), School of Electrical and Electronic \\ Engineering, Nanyang Technological University, Nanyang Avenue 639798, Singapore \\ (Received 9 March 2015; revised manuscript received 2 July 2015; published 8 September 2015)
}

\begin{abstract}
Plasmonic gratings that support both localized and propagating plasmons have wide applications in solar cells and optical biosensing. In this paper, we report on a most unusual grating designed to capture light efficiently into surface plasmons and concentrate their energy at hot spots where the field is resonantly enhanced. The dispersion of the surface plasmons shows degeneracy points at $k=0$, where, despite a strongly modulated grating, hidden symmetries forbid hybridization of plasmons traveling in opposite directions.
\end{abstract}

DOI: 10.1103/PhysRevX.5.031029

Subject Areas: Condensed Matter Physics,

Optics, Plasmonics

\section{INTRODUCTION}

Gratings diffract incident light, changing the photons' momentum parallel to the surface and creating several reflected rays. Here, we consider a class of gratings in which the period is much less than the wavelength of light. Although these gratings give rise to diffracted waves, they are all evanescent and stay close to the surface. Also, our gratings are assumed to be made of a metal with a plasmonic response, which we take to have the generic form

$$
\epsilon_{m}=1-\omega_{p}^{2} /[\omega(\omega+i \gamma)],
$$

with $\omega_{p}=8 \mathrm{eV}$ and $\gamma=0.032 \mathrm{eV}$.

Plasmonic gratings, in particular, have received increased attention in recent years, both in the context of plasmonic photovoltaics and in biosensing applications. The gratings' ability to diffract light and couple a plane wave into higher-order modes and their ability to confine light on a scale smaller than the wavelength is attractive. It has been exploited to enhance the photocurrent in and the absorptive properties of plasmonic solar cells $[1,2]$. The same properties are of interest in biosensing applications, where the strong-field confinement and enhancement aid detection of changes in the refractive index of the grating's environment. Several experimental and theoretical studies, which try to optimize the gratings' design to enhance sensing, exist [3-5]. Here, we propose a different method to study gratings based on transformation optics.

\footnotetext{
*matthias.kraft09@imperial.ac.uk

†luoyu@ntu.edu.sg
}

Published by the American Physical Society under the terms of the Creative Commons Attribution 3.0 License. Further distribution of this work must maintain attribution to the author(s) and the published article's title, journal citation, and DOI.
Transformation optics is a new technique that allows us to take a pattern of electric and magnetic fields and distort them as we please, returning through a simple formula the values of permittivity and permeability required if the distorted fields are to satisfy Maxwell's equations [6-10].

It has been applied to reshaping negatively refracting lenses and to designing cloaks of invisibility [11,12]. A particularly simple example is a two-dimensional conformal transformation that leaves the values of $\epsilon$ and $\mu$ unchanged in the two-dimensional plane [13]. Here, we start from a planar waveguide and apply a periodic conformal transformation to generate a grating structure. If we assume that the period of the grating is very short relative to the free-space wavelength and that the electric fields lie normal to the lines of the grating, then the fields are dominated by electrostatics.

This gives rise to very strong Fano-like plasmonic resonances in the response [14-17]. At Fano resonances, light is trapped and scattering reduced, allowing us to concentrate the optical energy into a periodic array of hot spots as if the resonant energy propagating along the surface has to squeeze itself into narrow gaps engineered into the grating, so greatly increasing the energy density. Because the grating has a very short period, these hot spots have a high density and therefore constitute an efficient means of enhancing nonlinear phenomena.

\section{GENERATING A PERIODIC GRATING FROM A SIMPLE SLAB}

Transformation optics derives its power from its ability to establish relationships between seemingly unrelated structures. This ability is best harnessed if it establishes relationships between complicated and simpler, i.e., more symmetric, structures. In this case, the symmetry of the simple structure and its relation to the more complicated 
one can be exploited to solve or simplify a physical problem, which one seeks to solve. References [18-24] provide successful examples, in which transformation optics has been used to solve various problems in plasmonics.

In this section, we show how we can relate a metallic periodic grating (in two dimensions) to a simple metal slab. We start from a simple infinite slab at position $x=x_{0}$ and with thickness $d$. The permittivity of the slab is given by $\varepsilon_{m}$. For simplicity, we assume that the permittivity of the surrounding medium is the same on both sides, denoted by $\varepsilon_{d}$, but this is not a necessary restriction. In the following, we will refer to this system as virtual space or slab frame.

The conformal transformation

$$
w=\gamma \log \left(\frac{1}{e^{z}-i w_{0}}+i y_{0}\right)
$$

with $z=x+i y$ and $w=u+i v$, maps the infinite slab to a periodic grating, as depicted in Fig. 1. $\gamma$ determines the overall dimension of the structure, while $w_{0}$ determines the modulation depth; $y_{0}$ is given by $y_{0}=\left[w_{0} /\left(e^{2\left(x_{0}+d\right)}-w_{0}^{2}\right)\right]$, and both $y_{0}$ and $w_{0}$ are real and positive.

It is important to note that the in-plane components of the permittivity and permeability are conserved under conformal maps [13]. Thus, the periodic grating possesses exactly the same in-plane material properties as the slab.
The coordinate system of the periodic grating is henceforth referred to as physical space or grating frame.

To clarify the effect of the transformation in Eq. (2), we present the analytical structure of it as a contour plot in Fig. 2. Starting from a regular Cartesian mesh, we can imagine the effect of any coordinate transformation as a pulling and stretching of the lines of constant $x$ and $y$ to distort the original mesh. This is exactly what is shown in Fig. 2: A regular Cartesian mesh is transformed to the irregular "grid" given in the figure. There are a number of points to note here. First, the contour lines cross at right angles, confirming that the transformation is conformal [25]. Second, the points where the contour lines "bunch up" correspond to points at infinity in the waveguide frame, which map into a periodic array of singularities, which are the branch points of the transformation. Associated with the branch points are the branch cuts running from the branch points to infinity (dashed lines). Last, if we follow a single contour line from the bottom to the top, we see that in between the branch points, this leads to a "wavy" contour line that repeats itself with period $2 \pi \gamma$. The modulation of this line gets stronger and stronger the closer to the branch points it is until, just at the branch point, it becomes infinitely modulated. Once we move past the branch points, something interesting happens. The contours, previously running continuously from bottom to top, split and form closed loops around the branch points. If we start in the slab
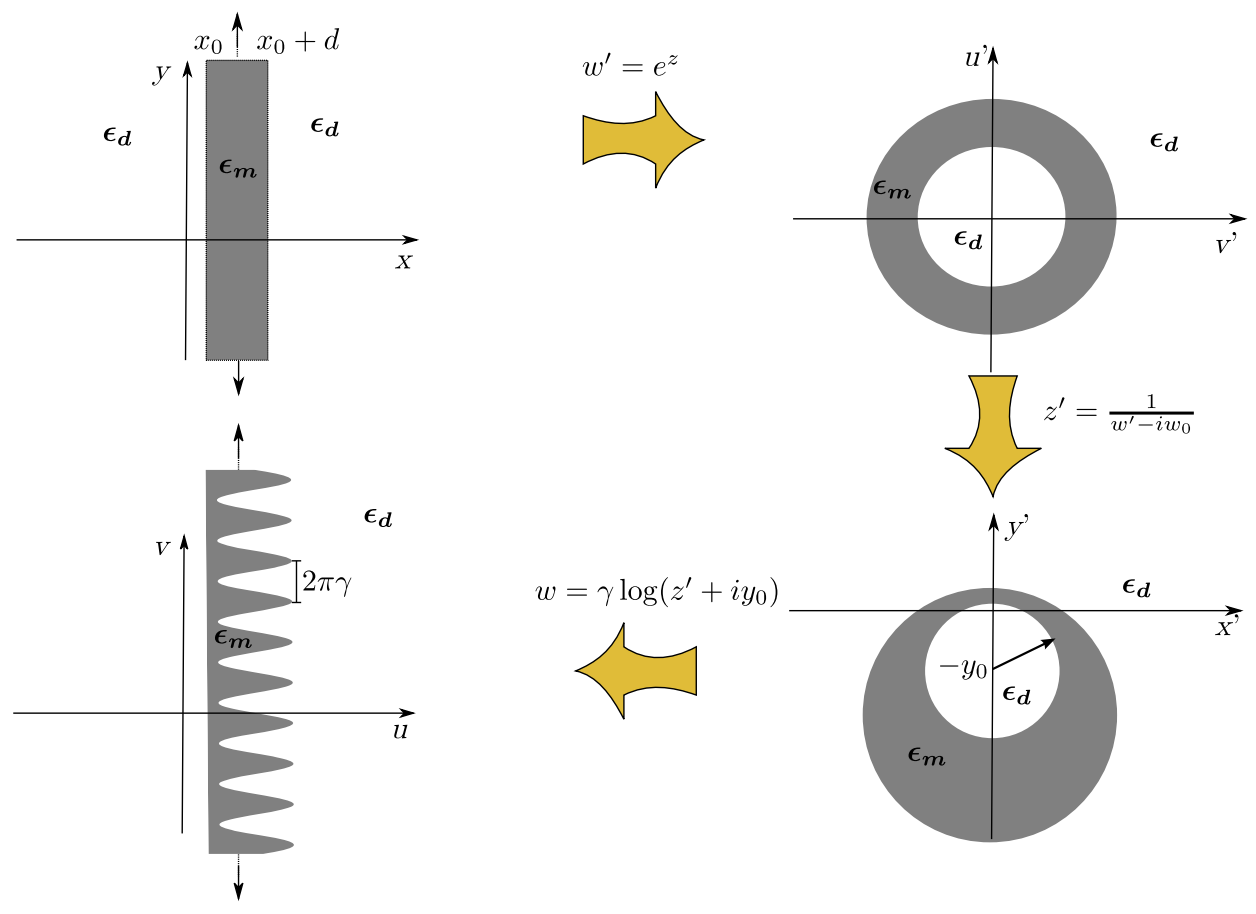

FIG. 1. Schematic of the transformations taking an infinitely long slab to a periodic grating. Each step gives the conformal transformation relating two neighboring structures. We use a complex number notation for the transformations, e.g., $w^{\prime}=u^{\prime}+i v^{\prime}$, $z=x+i y$, etc. An exponential map transforms a slab to an annulus, and an annulus is transformed to a nonconcentric annulus via a Möbius transformation [25]. Finally, a shifted logarithm takes a nonconcentric annulus to a grating with one flat and one modulated side. Note that $\gamma$ determines the periodicity and the overall dimension of the grating, while $w_{0}$ determines the modulation depth. 


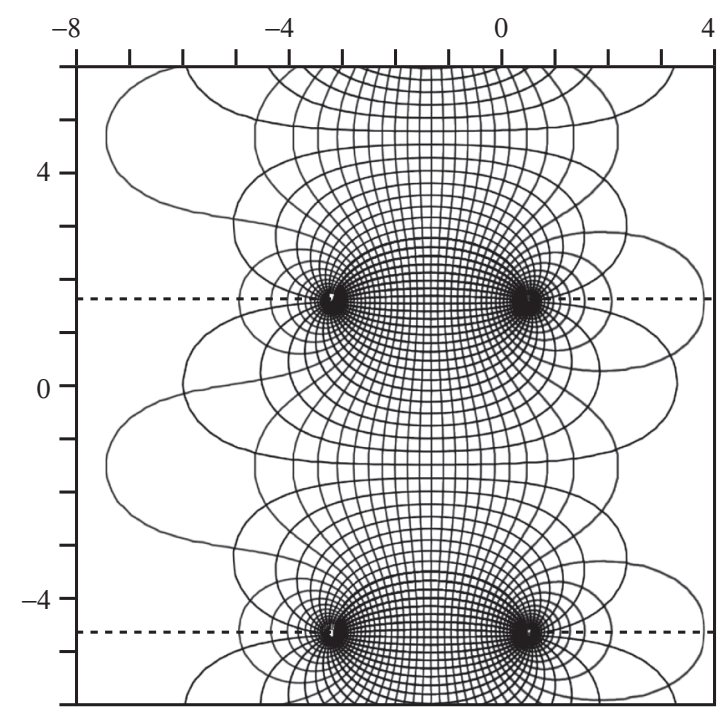

FIG. 2. Contour plot of the conformal transformation in Eq. (2). The solid lines show how the Cartesian coordinate grid in the $x, y$ frame is distorted under the transformation in the $u, v$ frame. The dashed lines show the branch cuts of the transformation running from the branch points to infinity. The parameters were $\gamma=1, y_{0}=0.033$, and $w_{0}=0.6$.

frame with a strip of metal bounded by lines of constant $x$ (see Fig. 1), this simple, translationally invariant planar structure is mapped into a periodically distorted grating. In the present paper, we restrict ourselves to the region between the branch points; the wavy contour lines essentially determine the surface of our grating, which we can modulate as strongly as we wish, by choosing the parameters of the transformation. However, outside this region, the closed loops around the branch points can be used to model periodically spaced nanoparticles.

The transformation really is very rich, not only allowing us to study periodically modulated gratings, but also nanoparticles on a substrate (see the Supplemental Material [26], Fig. S1), gratings with two modulated surfaces (by changing the position of a slab interface), half-planes with a corrugated interface (from a half-plane instead of a slab), and a chain of nanoparticles as in Refs. [27,28] (also from a half-plane). It relates a whole class of periodic gratings, which can be reverse-transformed into a simple slab. In the limit that electrostatics dominates the fields, the slab is translationally invariant and knows nothing of the grating's structure. However, magnetic effects do reflect the periodicity: The permeability that is assumed to be unity in the grating frame transforms in the slab frame to a periodic $\mu_{\perp}$. This residual magnetic component of the plasmons does sense the periodicity, but only weakly.

\section{ONE PLASMON RESONANCE CONDITION, A WHOLE CLASS OF GRATINGS}

One of the most fundamental characteristics of any plasmonic structure is its spectrum. In electrostatics, the spectral properties of a plasmonic structure are solely determined by its geometry and usually classified in terms of symmetries of said structure. Recently, it was shown that transformation optics can be used to reveal "hidden" symmetries not obvious at first sight [18]. This is because conformal transformations, apart from the in-plane permittivity and permeability, also conserve the electrostatic potential [29]. This well-known but nontrivial property of conformal transformations means that we can treat the slab system and grating frame as equivalent in the electrostatic limit, such that finding the plasmon modes and resonance condition in one frame automatically yields the ones in the other frame. The plasmon resonance condition of a translationally invariant slab of thickness $d$ is well known and given in Ref. [30]:

$$
e^{d\left|k_{y}\right|}= \pm\left(\frac{\varepsilon-1}{\varepsilon+1}\right)
$$

with $k_{y} \in \mathbb{R}_{0}$. By our argument above, this is also the plasmon resonance condition of a periodic grating generated from a slab of thickness $d$. (Formally, the plasmon resonance condition is obtained from the divergences of the expansion coefficients of the plasmon eigenmodes; see Supplemental Eq. S35.) Fixing the position and dimension of the slab, we can generate a whole class of gratings with different modulation depths and periods by tuning the two free parameters in Eq. (2): $w_{0}$ and $\gamma$. Each and every grating within that class derives its spectrum from the underlying slab structure, and we thus deem it appropriate to speak of a symmetry class. We must add two qualifications to this statement.

The first is to emphasize that it is true only in the electrostatic limit. Only the $x, y$ components of $\varepsilon$ and $\mu$ are invariant. This is not true of the perpendicular components, and even though our fields are predominantly electrostatic and contained in the $x, y$ plane, they will have small magnetic fields in the out-of-plane direction, which will interact with the transformation-induced periodic variation of $\mu_{\perp}$, which in the $x, y$ frame will spoil the perfect translational invariance enjoyed by the electrostatic fields.

The second and larger effect has to do with the boundary conditions that we must impose in the $x, y$ frame. If we consider a normally incident electromagnetic wave, wave vectors at the zone center are excited, which have the same periodicity as the grating in the $u, v$ frame. Thus, periodic solutions calculated in the $x, y$ are also periodic in the $u, v$ frame and give rise to no discontinuities at the branch cuts marked in Fig. 2. However, excitations corresponding to a finite Bloch wave vector may satisfy all conditions in the $x, y$ frame but now have an unphysical discontinuity of the phase across the branch cuts. Therefore, our statement of the equivalence of the slab structure to the grating only holds strictly at the zone center and approximately only in the vicinity of the zone center. However, illuminating the 


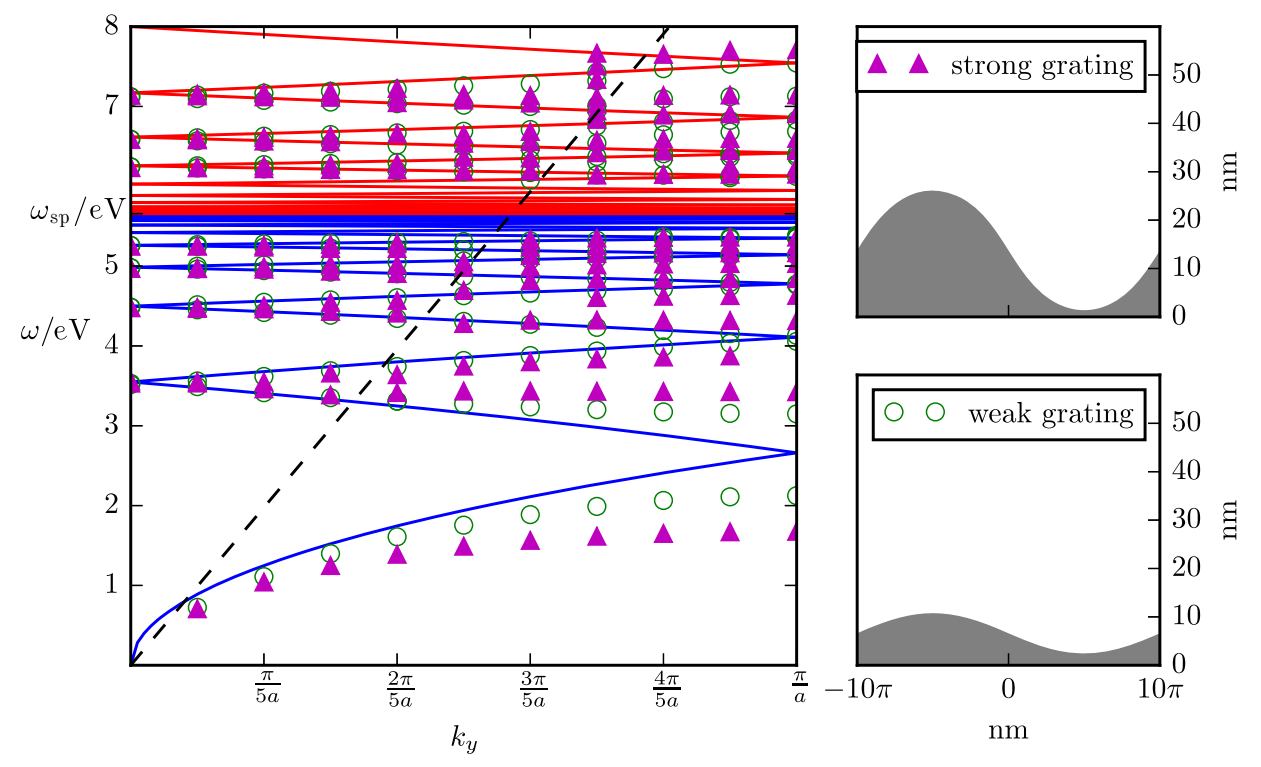

FIG. 3. Dispersion relation of two gratings in the first Brillouin zone. The magenta triangles and green open circles are COMSOL simulations for a grating with $\gamma=10^{-8}, w_{0}=2.5$ and $\gamma=10^{-8}, w_{0}=1.5$, respectively. $a=2 \pi \gamma$ in both cases, we start with a slab at position $x_{0}=1$ and with thickness $d=0.5$. The solid lines are analytical calculations. The dashed line corresponds to the light line.

grating with external radiation can couple only to states near the zone center, for we have assumed that the period of the grating is very small and hence $k_{0} \ll \pi / a$. This means that an external source of light excites only those modes that we can calculate accurately.

Figure 3 provides a test of this assertion. We compare the analytically derived band structure with the band structure for two different gratings obtained from full electrodynamic simulations (COMSOL). Both gratings are derived from the same slab. Note that Fig. 3 shows the dispersion relation in the first Brillouin zone. That means points at $k_{y}=k_{y}^{0}+$ $2 \pi * n / a$ on the dispersion curve $\omega\left(k_{y}\right)$ are folded back into the range 0 to $\pi / a$, i.e., the points $k_{y}=2 \pi / a, 4 \pi / a$, etc., are folded back onto the $k_{y}=0$ axis. For instance, the point at $\omega=3.5 \mathrm{eV}$ corresponds to the point $\omega\left(k_{y}=2 \pi / a\right)$ on the dispersion line and similarly for higher-order modes.

When $k_{y}=0$, we find near-perfect agreement between the analytical solution and the simulation. However, for larger values of $k_{y}$, the agreement gradually worsens, as the numerically obtained band structure features a large band gap at the zone edge. The physical reason for the band gap is Bragg scattering, as discussed in Ref. [31]. The disagreement is most prominent for the lowest energy mode, but is less severe for modes at higher energy. This is as predicted above due to problems with discontinuities across the branch cuts. However, again as predicted, solutions at the zone center are valid in both frames and the band gaps almost vanish for a planar structure as found in the $x, y$ frame (see Supplementary Fig. S2 for a magnified version of Fig. 3). Hence, the absence of gaps at the zone center is subject only to the qualification regarding magnetic effects, which give rise to small band gaps of the order of millivolts to tens of millivolts: a value which could be resolved in high-precision optical measurements in the absence of radiation damping but is hard to access in other experimental setups such as electron energy loss spectroscopy (despite recent progress in the field). This absent gap implies that we have found a "degeneracy point" at which two bands cross at $k=0$ but do not hybridize: The phase velocity goes to 0 for higher modes, but the group velocity remains finite. Strictly speaking, this holds only if the small magnetic repulsion between the bands can be ignored. As the magnetic repulsion leads to a small band gap, the dispersion relation becomes parabolic at $k_{y}=0$ but is linear for small $k_{y}$ [32].

The near-zero band gap for both gratings really is due to the hidden symmetry of the underlying slab structure. The results for small $k_{y}$ strengthen our belief that, in the electrostatic limit, a whole symmetry class of gratings can be generated from a single slab, thereby inheriting all the spectral properties that the symmetry of the slab imposes. This is the central result of this paper.

It is appropriate here to discuss the similarities and differences of our Dirac-like degeneracy points with actual "Dirac" points. The obvious similarity is the absence of an appreciable band gap at $k=0$, leading to a finite group velocity near the zone center. The emergence of Dirac points in graphene [33], photonic crystals [34], and their plasmonic analogue systems [35] is a direct consequence of a special symmetry of the underlying lattice (e.g., honeycomb lattice), leading to a conical dispersion relation around the Dirac point. We do not have a conical dispersion here, and the physical interpretation of the lack of band gap 
is a rather different one, too: There is no lattice, due to the translational invariance of the slab.

\section{CALCULATING THE OPTICAL RESPONSE OF THE GRATING UNDER PLANE-WAVE ILLUMINATION}

To illustrate the usefulness of the transformation optics approach in practical situations, we will calculate the optical response of two gratings belonging to the symmetry class, as discussed above. Specifically, we investigate transmission and reflection properties of the two gratings under plane-wave illumination with normal incidence. The two gratings are infinite along the vertical $(v-)$ direction and modulated on their right side. Their profiles are identical to the ones in Fig. 3. Here, we give the main results of the calculation; detailed derivations and definitions can be found in the Supplemental Material [26].

We consider the case of a plane-wave incident from the right and given by

$$
\begin{aligned}
\mathbf{H}^{\mathrm{sou}} & =-\frac{\omega \varepsilon \varepsilon_{0}}{k} E^{\mathrm{sou}} e^{-i k u \hat{z}} \\
& \approx-\frac{\omega \varepsilon \varepsilon_{0}}{k} E^{\mathrm{sou}}(1-i k u) \hat{z},
\end{aligned}
$$

where the approximation holds in the vicinity of the grating as long as the maximum thickness of the grating is much smaller than the wavelength of the incident radiation. Similarly to the electrostatic potential, the $z$ component of the $\mathbf{H}$ field is conserved under conformal transformation, as can be easily shown from the transformation formulas in Refs. [10,12]. Exploiting this property and the Fourier expansion of the coordinate transformation given in Eqs. S2 and S3 of the Supplemental Material [26], we can show that the incident potential in the slab frame is given by (Eq. S13)

$\phi^{\mathrm{sou}}=E^{\mathrm{sou}}\left[\sum_{\substack{g=-\infty \\ g \neq 0}}^{\infty} i \operatorname{sgn}(g)\left(d_{g}^{+} e^{|g| x}-d_{g}^{-} e^{-|g| x}\right) e^{i g y}+y\right]$

for $\log \left(w_{0}\right)<x<\log \left(w_{0}+1 / y_{0}\right)$. Inspection of the source potential in the slab frame shows that a plane wave in the grating frame, which only contains the zeroth-order Fourier mode, is transformed to a "wave" containing all higher-order modes in the slab frame. Vice versa, we might expect that each Fourier mode in the slab frame can couple to the zeroth-order mode in the grating frame under the reverse transformation. This coupling is crucial for the determination of the grating's radiative reaction. With the source potential known, we can write down the total potential in the regions of interest:

$$
\phi= \begin{cases}\phi_{L}=\phi^{\mathrm{sou}}+\phi_{L}^{\mathrm{near}}+\phi_{L}^{\mathrm{rad}} & \log \left(w_{0}\right)<x<x_{0} \\ \phi_{I}=\sum_{g=-\infty}^{\infty}\left(c_{g}^{-} e^{i g y} e^{-|g| x}+c_{g}^{+} e^{i g y} e^{|g| x}\right)+E_{0}^{v} y & x_{0}<x<x_{0}+d \\ \phi_{R}=\phi_{R}^{\text {near }}+\phi_{R}^{\mathrm{rad}} & x_{0}+d<x<\log \left(w_{0}+1 / y_{0}\right) .\end{cases}
$$

The near-field part of the potential $\phi_{R / L}^{\text {near }}$ describes the grating's ability to couple a plane wave into higher-order Fourier modes. It consists of evanescent waves $\left(e^{i g y} e^{ \pm|g| x}\right)$, which carry no energy to infinity. The radiative part $\phi_{R / L}^{\mathrm{rad}}$ incorporates information about the radiative reaction of the grating and is not present in a purely electrostatic calculation. The functional form of $\phi_{R / L}^{\text {rad }}$ can be obtained from the following simple argument. In the grating frame, the incident wave is normally incident and polarized along the $v$ axis. Since the grating is infinite along $v$, the plane wave leads to a potential drop or increase along that axis and the free electrons of the metal will be set in motion to produce a finite current. As this current can flow and oscillate on a scale larger than the wavelength of the incident radiation, it will itself lead to a scattered wave. The form of this scattered wave can be approximately taken as a plane wave at the frequency of the incident radiation. This approximation is reasonable as long as the grating is very "thin" because then only the currents along the direction where the grating is infinite contribute to the radiation and the grating can be taken as a thin current sheet. So the reflected and transmitted waves take the form [36]

$$
\begin{gathered}
\mathbf{H}^{\mathrm{ref}}=\frac{\omega \varepsilon \varepsilon_{0}}{k} E^{\mathrm{ref}} e^{i k u} \hat{z}, \\
\mathbf{H}^{\mathrm{tra}}=-\frac{\omega \varepsilon \varepsilon_{0}}{k} E^{\mathrm{tra}} e^{-i k u} \hat{z},
\end{gathered}
$$

where $E^{\text {ref }}$ and $E^{\text {tra }}$ have to be determined from the boundary conditions and are related to the total current along the $v$ direction in the grating. This means $\phi_{R / L}^{\text {rad }}$ take exactly the same form as the source potential in Eq. (6).

Applying the usual boundary conditions, i.e., demanding continuity of the normal component of the electric displacement field and the tangential component of the electric field at the interface, leads to a set of $4 G+2$ equations ( $G$ is the highest Fourier mode). However, there are $4 G+3$ unknowns (see the Supplemental Material, Eqs. S25-S30), 
one too many. This comes about because a purely electrostatic treatment does not contain any information on the radiative reaction, i.e., on $E^{\text {ref }}$ and $E^{\text {tra }}$. To determine $E^{\text {ref }}$ and $E^{\text {tra }}$ unambiguously, we need to introduce an additional "radiation" boundary condition. We obtain the form of the zeroth-order reflected and transmitted fields by approximating the grating as a current sheet. Maxwell's equations demand that the tangential component of the $\mathbf{H}$ field is discontinuous across a 2D current sheet, with the discontinuity given by the surface current [37]. This will be our additional boundary condition with the surface current given by the total current in the grating along the vertical direction. The total current in the grating can be calculated (see Supplemental Eq. S48) as $J=\gamma^{2} 2 \pi i \omega(\varepsilon-1) \varepsilon_{0}$ $\left(E^{\text {ref }}+E^{\text {sou }}\right) N$, with

$$
\begin{aligned}
N= & \sum_{\substack{g=-\infty \\
g \neq 0}}^{\infty}\left\{2 | g | ^ { 2 } \left[\frac{h_{g}^{+} c_{2,-g}^{+}}{2|g|}\left(e^{2|g|\left(x_{0}+d\right)}-e^{2|g| x_{0}}\right)\right.\right. \\
& \left.\left.-\frac{h_{g}^{-} c_{2,-g}^{-}}{2|g|}\left(e^{-2|g|\left(x_{0}+d\right)}-e^{-2|g| x_{0}}\right)\right]\right\}-d .
\end{aligned}
$$

Note that each of the higher-order Fourier modes contributes to the current in the grating. This proves our earlier statement that the higher-order Fourier modes in the slab frame can couple to the zeroth-order mode in the grating frame and vice versa. Combining the expression for the current with the boundary conditions allows us to unambiguously determine all scattering coefficients. This in turn allows us to write down expressions for the reflection and transmission coefficients:
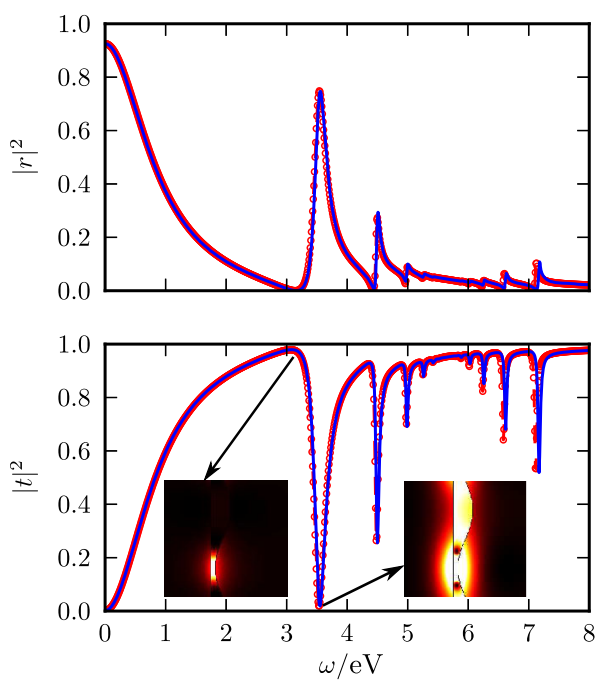

$$
\begin{aligned}
& r=\frac{i k\left(\varepsilon_{m}-1\right) \gamma N}{2-i k\left(\varepsilon_{m}-1\right) \gamma N}, \\
& t=\frac{2}{2-i k\left(\varepsilon_{m}-1\right) \gamma N} .
\end{aligned}
$$

The form of reflection and transmission coefficients is analogous to that of a $2 \mathrm{D}$ conductive sheet with surface conductivity $\sigma_{\text {eff }}=-i k\left(\varepsilon_{m}-1\right) \gamma N / Z_{0}$, where $Z_{0}$ is the impedance of free space. This indicates that the grating can be modeled as a 2D current sheet with an effective surface conductivity [38].

\section{REFLECTION AND TRANSMISSION PROPERTIES}

In this section, we compare our analytical results based on Eqs. (12) and (13) to full electrodynamic simulations using COMSOL. Figure 4 shows the reflectance and transmittance for the two gratings under plane-wave incidence. Here, we compare our analytical results with COMSOL simulations where the plane wave is incident from the right, i.e., on the modulated side of the grating. We can report an almost perfect agreement between analytics and simulations for the weakly modulated grating. There are only slight discrepancies in the height of the peaks in the transmittance spectrum. Note that the transmittance is close to unity around $3 \mathrm{eV}$, a feature shared by the more strongly modulated grating. Another feature shared by the two gratings is the position of the resonance peaks and the excellent agreement between analytics and simulations. In light of our earlier discussion of the band structure (see Fig. 3), this should not come as a surprise; however, it
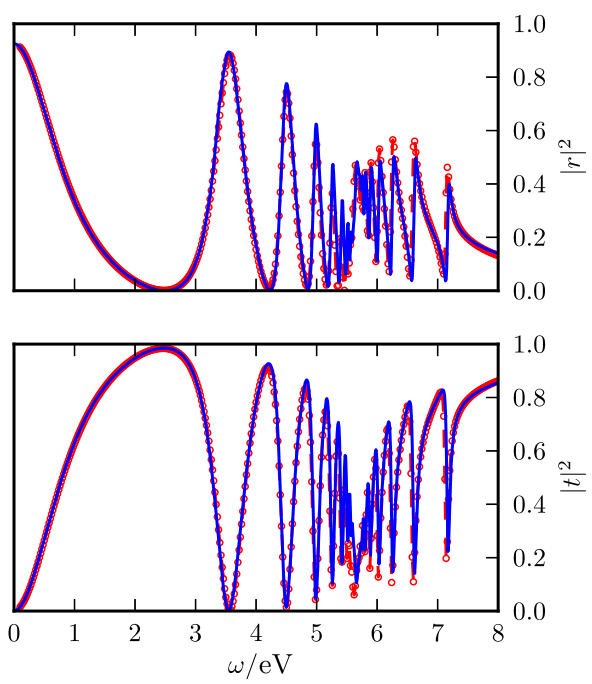

FIG. 4. Reflectance (top) and transmittance (bottom) for the weakly (left) and strongly (right) modulated gratings (shown in Fig. 3. Blue lines correspond to analytical calculations, whereas the red lines with circles are COMSOL simulations. In COMSOL, the wave is incident on the modulated side of the grating. The insets show the electric energy density in and close to the grating at the first transmission peak and dip (same color scale). For both analytics and simulations, the permittivity of the grating is $\varepsilon_{m}=$ $1-\omega_{p}^{2} /[\omega(\omega+i \gamma)]$ with $\omega_{p}=8 \mathrm{eV}$ and $\gamma=0.032 \mathrm{eV}$. The surrounding medium has $\varepsilon_{d}=1$. 
consolidates our earlier statement that in the quasistatic limit, the spectral properties of our special class of gratings are solely determined by the underlying slab structure.

Another common feature between the weakly and strongly modulated gratings that deserves mention is their ability to couple to the zeroth-order mode. As we can see in both spectra, there is a peak at zero frequency corresponding to the $g=0$ mode. This mode has not been observed in previous studies applying transformation optics to plasmonics such as Refs. $[18,20,21]$. The difference between the systems studied in, e.g., Refs. [18,20,21], and the grating is that the grating supports a continuous current on a scale larger than the wavelength. This leads to the coupling to the zeroth-order mode.

A closer look at the reflection spectra in Fig. 4 shows that the resonances have an asymmetric line shape akin to a Fano resonance [14-17] for the weakly modulated grating. Again, this can be understood via a conformal map. Figure 1 shows a relation between the grating and an asymmetric annulus; this structure has been shown to support Fano resonances, and a detailed study can be found in Ref. [21]. In agreement with the study in Ref. [21], the insets in Fig. 4 show that the electric energy is much more concentrated near the grating at the transmission dip than at the transmission peak. We attribute the considerably more symmetric line shapes for the strongly modulated grating to the stronger radiative damping.

Figure 4 also points toward the limitations of our approach. While the agreement between numerical simulations and analytics is near perfect for the weakly modulated grating, there are discrepancies for the higherorder modes (around $5.7 \mathrm{eV}$ ) of the strongly modulated grating. These discrepancies are likely to become worse for even stronger modulations. The fault lies with our assumption that the grating can be approximated as a current sheet radiating symmetrically on both sides. For strongly modulated gratings, this is not the case. We only mention here that this is due to the asymmetry of the grating, which means it acquires a bianisotropic character [39]; we leave a fuller discussion of this for a future study.

\section{FIELD DISTRIBUTION}

Of particular interest for applications such as Surfaceenhanced Raman spectroscopy (SERS) [40-42] is the electric field distribution and the field enhancement compared to the incident field. Figure 5 shows the imaginary part of the electrostatic potential for the plasmon resonance at approximately $4.5 \mathrm{eV}$. The results indicate that both the strongly and weakly modulated gratings, the mode is strongly concentrated in the proximity of the thinnest point of the grating. This is easily understood from the transformation used to generate the gratings. The slab from which we derive the gratings possesses a mirror plane along its vertical axis; thus, any plasmon mode excited in the slab will either be symmetric or antisymmetric across that axis.

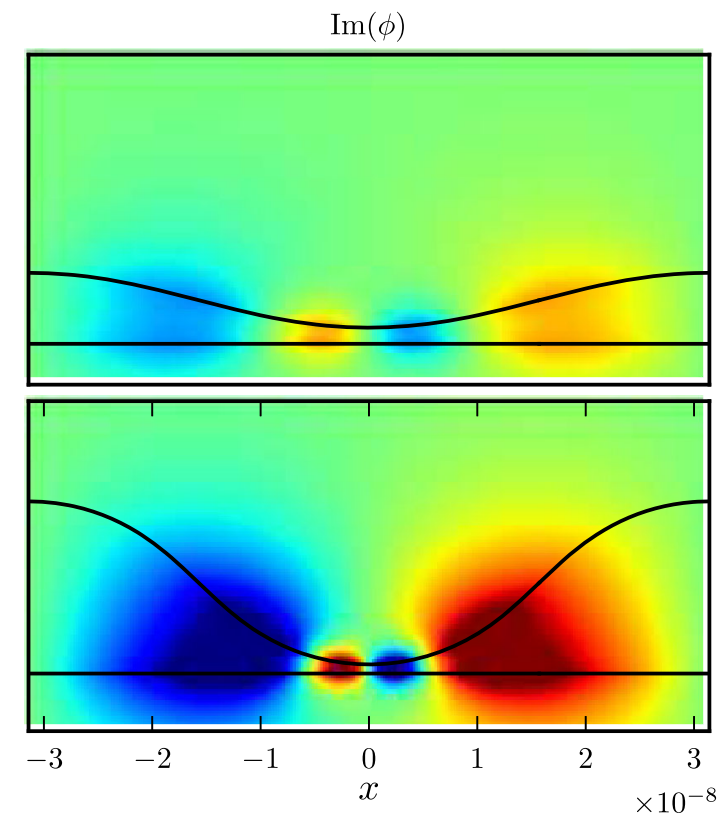

FIG. 5. Mode profile of the plasmon resonance at approximately $4.5 \mathrm{eV}$. We show the imaginary part of the electrostatic potential for the weakly modulated grating (top) and for the strongly modulated one (bottom). The color scales and the incident field are the same in both cases. The distributions have been obtained from the transformation optics approach using magnetic sources as described in the Supplemental Material [26]. We used the Drude model given in Eq. (1) for the permittivity. $\operatorname{Re}\left(\varepsilon_{m}\right)=-2.16$ and $\operatorname{Im}\left(\varepsilon_{m}\right)=0.022$ at $\omega \approx 4.5 \mathrm{eV}$.

Since the slab is invariant along the vertical direction and from Eq. (6), we know that each mode will also be periodic along the vertical direction and the energy spread out evenly. Figure 2 then explains why the electric fields are concentrated around the thinnest points of the grating. Near those points, the contour lines lie very dense in Fig. 2, indicating that space is strongly compressed in those regions. Thus, we should expect a very high energy density there. Likewise, near the thicker regions of the grating, space has been stretched and the energy spread out over a larger region. The stronger the modulation of the grating, the stronger this effect will be, as can be seen in Figs. 5 and 6. Our gratings thus provide an efficient way to funnel energy into a periodic array of hot spots. Just how strong these hot spots are can be seen in Fig. 6. Please note that in Fig. 6, we use experimental values of silver for the permittivity of the grating to obtain more realistic estimates of the field enhancement: $\operatorname{Re}\left(\varepsilon_{m}\right)=-3.96, \operatorname{Im}\left(\varepsilon_{m}\right)=$ -0.16 at $\omega \approx 3.18 \mathrm{eV}$ [43]. We can see that for both the weakly and strongly modulated gratings, the electric field strength is enhanced manifold $(\times 20-30)$ compared to the incident field. However, it is not quite as spectacular as for, e.g., two kissing cylinders [20]. A recent study [44], in which a grating with a period of $330 \mathrm{~nm}$ has been designed for SERS sensing, also showed a higher field enhancement (approximately 200). Yet, the gratings in this paper have 


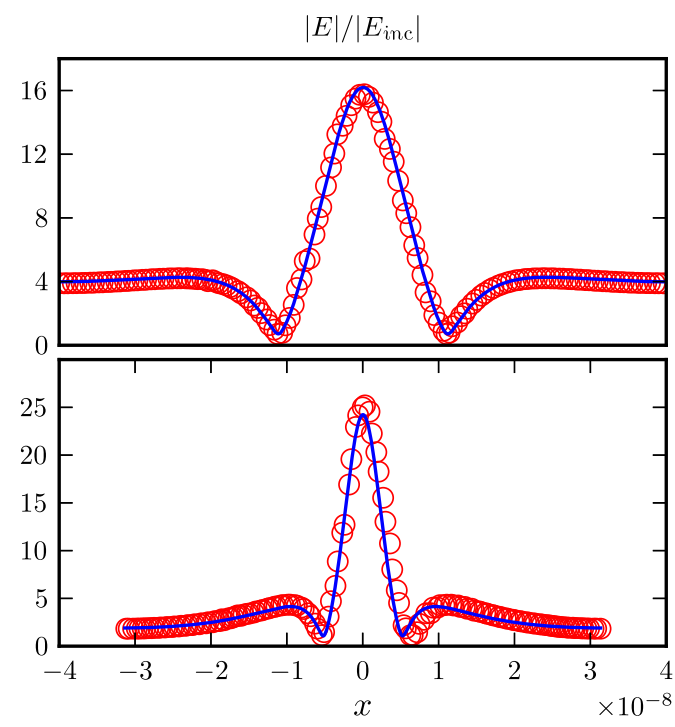

FIG. 6. Maximum field enhancement normalized by the incident electric field for the weakly (top) and strongly (bottom) modulated gratings. The data shows a 1D slice through the grating parallel to the $x$ axis. The $y$ position is such that the cut line is just below the lowest point of the modulated interface, i.e., always inside the grating. The red open circles show COMSOL simulation for a plane-wave incident on the modulated interface, and the blue solid lines are analytical calculations based on the transformation optics approach. In both cases, we use permittivity data of silver [43] with $\operatorname{Re}\left(\varepsilon_{m}\right)=-3.96$ and $\operatorname{Im}\left(\varepsilon_{m}\right)=-0.16$ at $\omega \approx 3.18 \mathrm{eV}$.

one big advantage over the two examples given above: a very high density of hot spots. We discuss a strongly subwavelength grating with period of approximately $63 \mathrm{~nm}$ leading to a great many hot spots on a very small area, giving a much higher chance that a target molecule will be located close to a hot spot. There is also a lot of room for improvement in the field enhancement. Our structure is derived from a nonconcentric annulus without singularity. The absence of a singularity reduces the field enhancement observed for these structures, compared to singular crescents [30]. However, our transformation optics approach is easily adapted to transform singular crescents into periodic gratings, in which case we would expect similar field enhancements as in Ref. [30].

\section{CONCLUSION}

In this paper, we gave another instance where transformation optics was able to reveal a hidden symmetry in plasmonic systems. Specifically, we showed how a conformal map can be used to generate periodic gratings from a simple slab. The invariance of the electrostatic potential under conformal maps implied that the periodic grating would inherit the spectral properties of the underlying slab structure. By tuning a free parameter of the transformation, we were thus able to generate a whole symmetry class of gratings, inheriting their spectral properties from the same slab, thus leading to degeneracy points at $k=0$. It is our belief that other plasmonic and dielectric gratings can be designed using our approach, insofar that they can be derived from a translationally invariant slab.

We further studied the optical response of these gratings under plane-wave illumination and compared with numerical simulations. We found that the weakly modulated grating supported Fano resonances. The agreement between analytics and numerics was excellent, and we showed that the gratings could be modeled as thin current sheets with effective surface conductivities. The "current sheet" model does, however, have its limitations, as it can only radiate symmetrically to both sides. For strongly modulated gratings, this becomes an issue for higher-order modes, as the strong asymmetry leads to an asymmetric radiation and the grating obtains a bianisotropic character. The detailed theoretical and experimental study of this bianisotropic effect will form the starting point for future investigations.

Furthermore, we discussed the electric field distributions and enhancements in the grating. While the field enhancements were lower than in previous studies in the literature, we believe that the gratings studied here could still provide a viable SERS sensing platform due to the high density of hot spots. Additionally, the simplicity of our analytical approach allows to easily optimize the grating's parameter if an optimization criterion is defined for an application at hand.

\section{ACKNOWLEDGMENTS}

We are thankful for fruitful discussions with Rongkuo Zhao and Paloma A. Huidobro. We acknowledge support from the Leverhulme Trust, the Gordon and Betty Moore foundation, MOE AcRF Tier 2, and NTU-A*start Silicon Technologies of Excellence under Grant No. 11235150003.

[1] J. N. Munday and H. A. Atwater, Large Integrated Absorption Enhancement in Plasmonic Solar Cells by Combining Metallic Gratings and Antireflection Coatings, Nano Lett. 11, 2195 (2011).

[2] L. Wen, F. Sun, and Q. Chen, Cascading Metallic Gratings for Broadband Absorption Enhancement in Ultrathin Plasmonic Solar Cells, Appl. Phys. Lett. 104, 151106 (2014).

[3] U. Bog, K. Huska, F. Maerkle, A. Nesterov-Mueller, U. Lemmer, and T. Mappes, Design of Plasmonic Grating Structures towards Optimum Signal Discrimination for Biosensing Applications, Opt. Express 20, 11357 (2012).

[4] A. Dhawan, M. Canva, and T. Vo-dinh, Narrow Groove Plasmonic Nano-Gratings for Surface Plasmon Resonance Sensing, Opt. Express 19, 787 (2011).

[5] W.-K. Kuo, C.-H. Chang, C.-J. Wu, and H.-H. Yu, PhaseDetection-Sensitivity Enhancement of Grating-Coupled Surface Plasmon Resonance Sensor with Light Incident at Nonzero Azimuth Angle, J. Nanophoton. 6, 063524 (2012). 
[6] H. Y. Chen, C. T. Chan, and P. Sheng, Transformation Optics and Metamaterials, Nat. Mater. 9, 387 (2010).

[7] A. J. Ward and J. B. Pendry, Refraction and Geometry in Maxwell's Equations, J. Mod. Opt. 43, 773 (1996).

[8] J. B. Pendry, A. Aubry, D. R. Smith, and S. A. Maier, Transformation Optics and Subwavelength Control of Light, Science 337, 549 (2012).

[9] Y. Luo, R. Zhao, A. I. Fernandez-Dominguez, S. A. Maier, and J. B. Pendry, Harvesting Light with Transformation Optics, Sci. China Inf. Sci. 56, 1 (2013).

[10] U. Leonhardt and T. G. Philbin, Transformation Optics and the Geometry of Light, Prog. Opt. 53, 69 (2009).

[11] U. Leonhardt, Optical Conformal Mapping, Science 312, 1777 (2006).

[12] J. B. Pendry, D. Schurig, and D. R. Smith, Controlling Electromagnetic Fields, Science 312, 1780 (2006).

[13] J. B. Pendry and S. A. Ramakrishna, Near-Field Lenses in Two Dimensions, J. Phys. Condens. Matter 14, 8463 (2002).

[14] Y. Francescato, V. Giannini, and S. A. Maier, Plasmonic Systems Unveiled by Fano Resonances, ACS Nano 6, 1830 (2012).

[15] B. Luk'yanchuk, N. I. Zheludev, S. A. Maier, N. J. Halas, P. Nordlander, H. Giessen, and C. T. Chong, The Fano Resonance in Plasmonic Nanostructures and Metamaterials, Nat. Mater. 9, 707 (2010).

[16] C. Genet, M. P. van Exter, and J. P. Woerdman, Fano-Type Interpretation of Red Shifts and Red Tails in Hole Array Transmission Spectra, Opt. Commun. 225, 331 (2003).

[17] M. Sarrazin, J.-P. Vigneron, and J.-M. Vigoureux, Role of Wood Anomalies in Optical Properties of Thin Metallic Films with a Bidimensional Array of Subwavelength Holes, Phys. Rev. B 67, 085415 (2003).

[18] M. Kraft, J. B. Pendry, S. A. Maier, and Y. Luo, Transformation Optics and Hidden Symmetries, Phys. Rev. B 89, 245125 (2014).

[19] J. B. Pendry, A. I. Fernandez-Dominguez, Y. Luo, and R. Zhao, Capturing Photons with Transformation Optics, Nat. Phys. 9, 518 (2013).

[20] A. Aubry, D. Y. Lei, S. A. Maier, and J. B. Pendry, Interaction between Plasmonic Nanoparticles Revisited with Transformation Optics, Phys. Rev. Lett. 105, 233901 (2010).

[21] J. Zhang and A. V. Zayats, Multiple Fano Resonances in Single-Layer Nonconcentric Core-Shell Nanostructures, Opt. Express 21, 8426 (2013).

[22] P. A. Huidobro, M. L. Nesterov, L. Martín-Moreno, and F. J. García-Vidal, Transformation Optics for Plasmonics, Nano Lett. 10, 1985 (2010).

[23] Y. Liu, T. Zentgraf, G. Bartal, and X. Zhang, Transformational Plasmon Optics, Nano Lett. 10, 1991 (2010).

[24] Y. Luo, R. Zhao, and J. B. Pendry, van der Waals Interactions at the Nanoscale: The Effects of Non-locality, Proc. Natl. Acad. Sci. U.S.A. 111, 18422 (2014).

[25] M. J. Ablowitz and A.S. Fokas, Complex Variables: Introduction and Applications, Cambridge Texts in Applied Mathematics (Cambridge University Press, Cambridge, England, 2003).
[26] See Supplemental Material at http://link.aps.org/ supplemental/10.1103/PhysRevX.5.031029 for detailed mathematical derivations and complementing figures.

[27] R. C. McPhedran and D. R. McKenzie, Electrostatic and Optical Resonances of Arrays of Cylinders, Appl. Phys. 23, 223 (1980).

[28] R. C. McPhedran and G. W. Milton, Transport Properties of Touching Cylinder Pairs and of the Square Array of Touching Cylinders, Proc. R. Soc. A 411, 313 (1987).

[29] R. Schinzinger and P. A. A. Laura, Conformal MappingMethods and Applications (Dover, New York, 2003).

[30] A. Aubry, D. Y. Lei, S. A. Maier, and J. B. Pendry, Broadband Plasmonic Device Concentrating the Energy at the Nanoscale: The Crescent-Shaped Cylinder, Phys. Rev. B 82, 125430 (2010).

[31] W. L. Barnes, T. W. Preist, S. C. Kitson, and J. R. Sambles, Physical Origin of Photonic Energy Gaps in the Propagation of Surface Plasmons on Gratings, Phys. Rev. B 54, 6227 (1996).

[32] This is as clarified by one of the anonymous referees.

[33] G. W. Semenoff, Condensed-Matter Simulation of a Three-Dimensional Anomaly, Phys. Rev. Lett. 53, 2449 (1984).

[34] X. Huang, Y. Lai, Z. H. Hang, H. Zheng, and C. T. Chan, Dirac Cones Induced by Accidental Degeneracy in Photonic Crystals and Zero-Refractive-Index Materials, Nat. Mater. 10, 582 (2011).

[35] G. Weick, C. Woollacott, W. Barnes, O. Hess, and E. Mariani, Dirac-Like Plasmons in Honeycomb Lattices of Metallic Nanoparticles, Phys. Rev. Lett. 110, 106801 (2013).

[36] R. Feynman, R. Leighton, and M. Sands, The Feynman Lectures on Physics, 2nd ed. (Addison-Wesley, Boston, 1963), Vol. 2.

[37] J. D. Jackson, Classical Electrodynamics, 3rd ed. (Wiley, New York, 1998).

[38] P. Tassin, T. Koschny, and C. M. Soukoulis, Effective Material Parameter Retrieval for Thin Sheets: Theory and Application to Graphene, Thin Silver Films, and Single-Layer Metamaterials, Physica (Amsterdam) 407B, 4062 (2012).

[39] J. A. Kong, Electromagnetic Wave Theory, 6th ed. (EMW Publishing, Cambridge, Massachusetts, 2005).

[40] M. Moskovits, Surface-Enhanced Spectroscopy, Rev. Mod. Phys. 57, 783 (1985).

[41] A. Campion and P. Kambhampati, Surface-Enhanced Raman Scattering, Chem. Soc. Rev. 27, 241 (1998).

[42] J. B. Jackson and N. J. Halas, Surface-Enhanced Raman Scattering on Tunable Plasmonic Nanoparticle Substrates, Proc. Natl. Acad. Sci. U.S.A. 101, 783 (1972).

[43] P. B. Johnson and R. W. Christy, Optical Constants of the Noble Metals, Phys. Rev. B 6, 4370 (1972).

[44] X. Deng, G. B. Braun, S. Liu, P. F. Sciortino, B. Koefer, T. Tombler, and M. Moskovits, Single-Order, Subwavelength Resonant Nanograting as a Uniformly Hot Substrate for Surface-Enhanced Raman Spectroscopy, Nano Lett. 10, 1780 (2010). 